\title{
Perspectivas de la investigación en micología
}

\author{
TEÓFILO HERRERA \\ Departamento de Botánica, Instituto de Biología, UNAM. Apdo. Postal 70-233. 04510 México, D.F.
}

\begin{abstract}
Resumen. Se presenta un panorama sobre las posibilidades de investigación que hay en el campo de la micología. Se indican las vastas perspectivas que existen en este campo sin precisar muchos detalles sobre cada una de las grandes ramas de la micología dada la gran variedad de las mismas. Concretamente, se comenta sobre diversas perspectivas de investigación en las siguientes áreas: 1) taxonomía y biogeografía, 2) ecología y evolución, 3) biología celular, biología molecular y genética, 4) bioquímica, biofísica y fisiología, 5) biotecnología, 6) micología forestal y agrícola y fitopatología, 7) micología médica humana y veterinaria.
\end{abstract}

Abstract. A general view on the possibilities of research in the field of mycology is presented. The wide perspectives in this field are indicated avoiding many details about each of the big areas of mycology selected to cover this exposure. Comments are made summarizing on several perspectives of research in the following areas of science: 1) taxonomy and biogeography, 2) ecology and evolution, 3) cellular biology, molecular biology and genetics, 4) biochemistry, biophysics and physiology, 5) biotechnology, 6) forest and agricultural mycology and phytopathology, 7) human and veterinary medical mycology.

\section{INTRODUCCIÓN}

Como en otras ciencias, las perspectivas de investigación en micología pueden tener dos orientaciones fundamentales: las teóricas o básicas y las prácticas o aplicadas, aunque hay entre ambas estrechas conexiones, además de que las segundas necesariamente deben estar apoyadas sobre las primeras y es constante la retroalimentación entre ambas.

Muy diversos aspectos teóricos y prácticos de la micología han sido investigados hasta el momento, los cuales ofrecen siempre perspectivas de ser ampliados y profundizados en forma ilimitada, aun cuando algunos de ellos parezcan estar suficientemente estudiados. Hay varios libros que explican ya sea en forma sucinta o con detalle las áreas de estudio de la micología considerando diferentes enfoques que incluyen los de enseñanza e investigación. (Buller, 1909-1950; Alexopoulos y Mims, 1985; Herrera y Ulloa, 1990).

\section{ÁREAS DE ESTUDIO}

\section{Taxonomía y biogeografía.}

Una alta proporción de los trabajos que han sido realizados sobre micología corresponde a esta primera área, la que por otra parte casi siempre es la fase inicial de las investigaciones que se emprenden en los diversos campos de las ciencias naturales. Debido a esto, con frecuencia se considera que los estudios taxonómicos han quedado relegados a la biología tradicional ya establecida y que no tiene muchas posibilidades de profundizar en investigaciones que revelen aspectos novedosos y culminen en descubrimientos importantes. No obstante, la investigación taxonómica ofrece interesantes perspectivas a las generaciones actuales y futuras de micólogos, pues ambas tendrán la oportunidad de seguir el dinamismo de los cambios taxonómicos con base en nuevas técnicas. Por lo demás, muchas de éstas son complejas porque implican la participación interdisciplinaria y utilizan métodos muy especializados tomados de otras ciencias, por ejemplo, de la física y la química, de la biología celular y de la biología molecular. En esta forma se están obteniendo datos que modifican las clasificaciones y los conceptos taxonómicos sobre los hongos. Por tanto, la taxonomía no sólo sería una fase inicial de la investigación taxonómica, sino también la culminación de la misma, una vez que se apliquen los conocimientos obtenidos por los métodos modernos y los que se desarrollen en épocas futuras (Talbot, 1971).

La investigación sobre la biodiversidad, que tanto interés ha despertado en la actualidad no sólo entre los biólogos sino en muy diversos niveles de las actividades profesionales y gubernamentales, es un vasto campo de trabajo en lo que concierne a la investigación micológica. Baste considerar que el reino Fungi es uno de los más diversificados y versátiles que hay en la naturaleza, y que gran parte de sus representantes aún se desconocen debido a las escasas investigaciones sobre muchos de ellos, en particular los que hasta el momento no tienen una importancia práctica. Tal es el caso de los hongos que se desarrollan en los vastos ambientes acuáticos continentales y marinos. Los hongos marinos, por ejemplo, apenas se empezaron a estudiar a nivel mundial y en forma sistemática en la segunda mitad de este siglo y, en México, a partir de la última década, aunque algunos investigadores extranjeros ya habían hecho estudios esporádicos en este país tiempo atrás (Kohlmeyer y Kohlmeyer, 1979). Ahora bien, todos los animales y plantas están asociados con los hongos ya sea como saprobios, simbiontes o parásitos, a veces en forma permanente o transitoria con hongos endófitos o endozoicos 
y epífitos o epizoicos. Dado que un gran número de dichos hongos son selectivos de una determinada especie vegetal o animal, sería suficiente pensar en los hongos asociados con los insectos, que es el grupo de seres vivos que tiene el mayor número de especies registradas en la actualidad, para admitir que el número de especies de hongos en la naturaleza, aun considerando la ubicuidad de muchos de ellos, puede rebasar ampliamente la cifra de un millón. Esta cifra es mucho mayor que el rango100,000 a 200,000 que se ha considerado en épocas recientes. Por tanto, el trabajo taxonómico básico que se requiere para ampliar y profundizar el conocimiento de la diversidad fúngica necesita apoyo en forma prioritaria. Tal apoyo es una condición para llegar a conocer con cierta exactitud este campo de la micología que es la base del aprovechamiento de los recursos naturales correspondientes al importante grupo de hongos (Hawksworth et al., 1983; Ainsworth et al., 1994).

El estudio de la distribución de los hongos en las diversas regiones geográficas de nuestro planeta corresponde al área de la biogeografía, sobre la cual se han hecho numerosas investigaciones pero no en forma equilibrada. Por ejemplo, en algunos países europeos está bastante bien estudiada la flora micológica, ahora denominada micobiota, en tanto que en otros países, como es el caso de México, hay extensas regiones que han sido poco exploradas con el objetivo de conocer los hongos que se desarrollan en ellas. Hay que notar, sin embargo, que actualmente se hacen esfuerzos para impulsar este tipo de estudios con la tendencia de lograr la elaboración de inventarios de los recursos naturales, trabajo que seguramente se prolongará hacia los años futuros. Será de especial importancia la realización de inventarios sobre la diversidad de los hongos a nivel mundial y de diversos países en particular, lo cual ofrece un amplio campo de trabajo para los investigadores que se interesen en la micología. Por otra parte, los conocimientos adquiridos mediante estos estudios permitirán profundizar en la investigación de los factores que determinan las leyes de la distribución geográfica de las especies de hongos, en relación con las características ecológicas de los diversos ambientes que existen en la tierra, tanto en las regiones calientes como en las templadas y frías o en las regiones húmedas, áridas y desérticas. Además, la distribución geográfica de los hongos está íntimamente relacionada con la distribución de las plantas y de los animales, principalmente en el caso de los hongos simbióticos, comensales o parásitos. Por ejemplo, la distribución de algunos hongos micorrícicos estrictos sigue exactamente la distribución de sus plantas hospederas, como son los casos de muchas especies de coníferas, fagáceas y betuláceas en los que no pueden prosperar las plantas sin sus hongos simbióticos, ni éstos sin aquellas (Marks y Kozlowski, 1973). Lo mismo puede decirse de otros tipos de hongos como los parásitos o los que viven asociados en relaciones mutualistas con diversos tipos de animales, como los insectos.

\section{Ecología y evolución}

Es oportuno comentar que los hongos, como todos los seres vivos, tienen relaciones constantes con los ambientes en que se desarrollan, incluyendo las complejas interacciones de unos hongos con otros, con las moneras, los protistas, las plantas y los animales. Forman parte así de la intrincada red que se ha establecido en el equilibrio de la naturaleza. Muchas de estas interrelaciones son bien conocidas, pero otras deberán ser estudiadas en el futuro y, aun las que están bien definidas, como las de los hongos parásitos de plantas y animales y las de los hongos simbióticos formadores de micorrizas y líquenes, deberán ser estudiadas con enfoques fisiológicos, bioquímicos y genéticos más profundos. Ello permitirá explicar con una precisión cada vez mayor las causas íntimas que determinan dichas relaciones. Es obvio que la investigación taxonómica a nivel específico, infraespecífico, genérico y supragenérico es indispensable para conocer los mecanismos de adaptación a los diversos ambientes dentro de sus correspondientes nichos ecológicos, los cuales llegan a ser muy característicos y muy bien definidos para cada especie de hongo y para cada una de sus variedades y formas ecológicas. Estos estudios permitirán explicar sobre bases científicas bien definidas, lo concerniente a las posibles líneas evolutivas que han seguido los diferentes grupos de hongos hasta establecerse en la forma que se les conoce actualmente. El problema de las líneas evolutivas se dificulta por la escasez de hongos fósiles en los registros geológicos, y por la discrepancia que existe en los criterios y las especulaciones de los micólogos para explicar la evolución de los diversos grupos de hongos y sus relaciones filogenéticas; por este motivo, en el futuro pueden cambiar mucho los fundamentos científicos que actualmente intentan explicar los fenómenos evolutivos de los hongos (Robinson, 1967; Raper y Flexer, 1971).

\section{Biología celular, biología molecular y genética}

En lo que concierne a la micología, sólo una proporción pequeña de las especies conocidas de hongos ha sido estudiada con detalle en relación a estas tres áreas del conocimiento. De tal manera, hay mucho trabajo por delante, con las atractivas posibilidades de correlacionar los conocimientos adquiridos utilizando hongos como material de trabajo, con los descubrimientos logrados en otros tipos de organismos, pues muchos fenómenos involucrados en las áreas mencionadas son semejantes en todos los seres vivos.

Se conocen las características celulares fundamentales de la mayor parte de los hongos, y un buen número de éstos ha sido estudiado con detalle con la ayuda del microscopio óptico y en menor proporción utilizando microscopios electrónicos tanto de barrido como de transmisión. Esto ha permitido describir con detalle las estructuras celulares. Habrá que seguir desarrollando este proceso, en particular con las numerosas especies poco estudiadas, las recientemente descritas o las que están por ser descubiertas. 
Al profundizar en el estudio citológico de los hongos se puede pasar insensiblemente al campo de la biología molecular de los mismos. Así ha sucedido, por ejemplo, en las investigaciones sobre la formación de la pared celular de algunos hongos mucoráceos, caso en el que al mismo tiempo se precisan las características de la pared celular, la naturaleza química y la distribución arquitectónica de las moléculas que constituyen dicha estructura. Este tipo de información también se ha obtenido o se pretende investigar detalladamente sobre las estructuras intracelulares, en particular la membrana citoplasmática, las mitocondrias, el aparato de Golgi, la membrana nuclear y los cromosomas. El estudio de estos últimos condujo al descubrimiento de las moléculas fundamentales para el mantenimiento, el control y la transmisión de la vida, que son las del ácido desoxirribonucleico (ADN). Estas moléculas constituyen a los genes, característicos de cada especie biológica y, por supuesto, de cada especie de hongo. No obstante, de muy pocas especies de hongos se tienen mapas cromosómicos completos. Son relativamente pocas las investigaciones que se han hecho para precisar en estos organismos el funcionamiento de los ácidos nucleicos en el control de los fenómenos de la herencia. En estas investigaciones, que corresponden al complejo tema de la genética de hongos, se han logrado cambios sorprendentes en las especies de hongos mediante las técnicas de la ingeniería genética actualmente en pleno desarro1lo. La genética de los hongos presenta muchas perspectivas de investigación debido a que son pocas las especies de hongos estudiadas desde esta perspectiva. Por ejemplo, se han realizado investigaciones en especies de los géneros Neurospora y Schizophyllum y en ciertas levaduras, como la de la cerveza (Saccharomyces cerevisiae). Estos estudios están en marcha por la facilidad de obtener cultivos de muchas generaciones de tales especies en muy poco tiempo (Ainsworth y Sussman, 1965; Fincham et al., 1979).

\section{Bioquímica, biofísica y fisiología}

Se han hecho estudios muy detallados sobre la constitución química de muchas especies de hongos. De otras especies sólo se han realizado análisis químicos generales que no alcanzan la precisión para registrar la presencia de la gran diversidad de compuestos que pueden formar parte de los hongos que representan a dichas especies. Además, de la mayoría de las especies de hongos no se ha hecho este tipo de estudios, aunque con muchos de ellos se han utilizado las técnicas generales para registrar la producción de antibióticos, enzimas y otras sustancias de posible aplicación médica $\mathrm{o}$ industrial. En el futuro seguramente continuarán las investigaciones sobre la bioquímica de los hongos, no sólo en el aspecto global o general sino tratando de localizar los compuestos químicos y la génesis de los mismos en cada una de las estructuras que constituyen a las diversas especies, lo que incluirá el análisis fino y detallado de las estructuras intracelulares y de las paredes celulares.
Las características biofísicas han sido mucho menos estudiadas que las bioquímicas. En muy pocas especies se han tomado las características físicas y fisicoquímicas: estado coloidal, conductividad eléctrica, potenciales de oxidorreducción, valores termoquímicos y termodinámicos, equilibrio iónico y $\mathrm{pH}$ de las distintas estructuras celulares, $\mathrm{pH}$ del medio en que se desarrollan los hongos, el efecto de la luz y otras radiaciones incluyendo las de los radioisótopos, las reacciones fotoquímicas y la bioluminiscencia. Aunque el estudio de estas características puede aplicarse a todos los seres vivos, los hongos constituyen un material relativamente fácil de manejar para los propósitos de la investigación biofísica.

El conocimiento de las características químicas, físicas y fisicoquímicas, a su vez, es muy importante en los diferentes enfoques de la investigación sobre la fisiología de los hongos, que ha sido muy estudiada y ofrece la utilización de los numerosos datos que ha obtenido a futuras investigaciones, más diversificadas y profundas. Por ejemplo, funciones de los hongos en relación con su ambiente, transferencia de energía y respiración, nutrición y movimiento del agua y los solutos en el protoplasma, síntesis de ácidos nucleicos, reproducción y mecanismos de dispersión, tropismos y tactismos. Por otra parte, en muchas especies de hongos estos fenómenos no han sido investigados o sólo se conocen de ellos algunos aspectos elementales como las estructuras de multiplicación vegetativa y de reproducción, de manera que hay un amplísimo campo por investigar con perspectivas de seguir importantes enfoques, incluyendo los de la micología aplicada (Buller, 1909-1950; Robinson, 1978).

\section{Biotecnología y micología industrial}

Existen amplias perspectivas dentro de esta área en relación con la investigación micológica actual, a partir de una visión retrospectiva de la participación de los hongos en las actividades humanas desde los tiempos más remotos de la historia, en los que el hombre disfrutó, entre otras cosas, de la cerveza, del vino y del pan. Estos productos se obtuvieron en forma empírica con la participación de las levaduras, las que espontáneamente transforman diversas materias primas en bebidas y alimentos fermentados que han tenido una repercusión importante en la historia de la humanidad. En la época actual es posible orientar y controlar la fisiología de las levaduras con fines industriales, por ejemplo, para metabolizar hidrocarburos y sintetizar altas cantidades de proteínas o determinadas vitaminas, en particular las del complejo B. Además de las levaduras, muchos otros hongos son también utilizados con los mismos propósitos o bien para producir diversas sustancias de importancia económica considerable como antibióticos, enzimas, ácidos orgánicos y esteroides. Todavía hay mucho que investigar para sacar el mayor provecho de las actividades metabólicas de los hongos lo cual incrementará la cantidad de importantes productos que se pueden obtener mediante el manejo biotecnológico adecuado de cepas seleccionadas de las especies 
involucradas en los procesos industriales.Así que se logrará un notable incremento de los productos industriales siguiendo las ingeniosas técnicas de la ingeniería genética, que ya se practican con éxito en la actualidad y ofrecen perspectivas de investigación cada vez más atractivas.

Además de los productos citados antes, se pueden obtener otros muchos en forma empírica o industrialmente, con las consecuentes ventajas económicas que en numerosos casos llegan a dar rendimientos multimillonarios cuando es posible manipular y controlar a los hongos de importancia industrial. Algunos de estos productos son: quesos madurados con hongos, como el roquefort, el danés azul y el camembert, que utilizan hongos del género Penicillium ( $P$. roquefortii, $P$. glaucum, P. camembertii).Hongos del mismogénero (P.notatum, $P$. chrysogenum) son seleccionados, después de amplias investigaciones micológicas y biotecnológicas, para producir penicilina, conocido antibiótico cuya demanda es enorme a nivel mundial. Otro antibiótico importante, la griseofulvina, es producido por un hongo del género antes citado ( $P$. griseofulvum), en tanto que los antibióticos del grupo de las cefalosporinas son producidos por hongos del género Cephalosporium ( $C$. acremonium). Lo importante es anotar, para los fines de esta referencia, que en la producción de antibióticos, igual que en la obtención de otras sustancias es necesario tener una base de investigación que garantice el éxito de la actividad industrial (Smith, 1960).

Otros productos que se obtienen industrialmente con la participación de los hongos y que ofrecen una problemática semejante a la anotada antes son los siguientes: grasas, alcoholes, alcaloides, pigmentos, bebidas y alimentos fermentados tradicionales de México, como el pulque, el pozol, el tesgüino, el colonche y el tepache, así como los tradicionales de otras partes del mundo, como la salsa de soya y diversos productos fermentados de soya o de arroz muy utilizados en Oriente, por ejemplo, en China, Japón e Indonesia. Se han hecho diversos estudios y se han proyectado investigaciones recientes para obtener el mayor rendimiento de muchos de estos productos, los cuales además ofrecen una atractiva fuente de investigaciones etnológicas.

Los hongos comestibles representan un campo más en las investigaciones biotecnológicas y etnológicas. El hombre los ha utilizado como alimento desde tiempos remotos, aunque frecuentemente con resultados negativos o sorprendentes, al confundirlos con hongos venenosos o alucinógenos.

La utilización de los hongos comestibles y la estimación de algunos de ellos como delicadezas culinarias, ha motivado al establecimiento de industrias que requieren procedimientos biotecnológicos sostenidos por una base de investigación minuciosa para lograr rendimientos satisfactorios en las plantas de producción, las cuales pueden llegar a proporcionar un importante rendimiento económico cuando se sigue el procedimiento científico adecuado (Martín et al., 1984).

El incremento de las investigaciones actuales sobre el cultivo de los hongos comestibles, abre amplias perpectivas para el cultivo de un gran número de especies y no sólo del hongo que tradicionalmente ha sido cultivado en muchas partes del mundo (Agaricus bisporus y otras especies del mismo género). Con frecuencia muchos hongos son utilizados como ingredientes fundamentales o como condimentos de platillos de elevado refinamiento gastronómico (Chang y Hayes, 1978).

En micología industrial es también importante el estudio del biodeterioro o biodegradación que pueden ocasionar muchos hongos en todas las materias primas y en los productos industriales. El control y combate de estos hongos es un campo de investigación que seguirá proporcionando las medidas más adecuadas para evitar el deterioro o la destrucción de materiales y productos de importancia económica. Cuando estos hongos contaminan alimentos, además de ocasionar la alteración de éstos, pueden producir micotoxinas de peligrosos efectos patológicos, como las aflatoxinas, las cuales tienen actividad cancerígena comprobada en el hombre y en varios animales domésticos (Christensen, 1975).

\section{Micología forestal, agrícola y fitopatología}

La presencia de los hongos en el suelo es indispensable para el buen desarrollo de las plantas, pues intervienen desde la génesis del suelo hasta la formación de las micorrizas que establecen una relación simbiótica facultativa u obligada en las raíces de la mayor parte de las especies vegetales, tanto herbáceas como arbustivas y arbóreas. Así que, sin los hongos, no existiría la gran diversidad de bosques y de otras formaciones vegetales que pueblan extensas áreas de nuestro planeta y que sostienenen una notable diversidad faunística. Estudiar y cuidar esta biodiversidad implica incursionar en atractivos e inagotables temas de investigación, un importante número de ellos con un enfoque micológico.

La actividad agrícola, igual que la forestal, también implica una buena relación de las plantas con los suelos donde se desarrollan, dependiendo el éxito de la producción agrícola, en gran parte, de la presencia de determinados hongos en esos suelos. Tal es el caso de los hongos biodegradadores y de los micorrícicos, sin descartar el importante papel de las bacterias y de otros microorganismos en los procesos de transformación de los materiales edáficos. Como en el caso de los aspectos forestales, la agricultura debe desarrollarse sobre la base de resultados de investigaciones multidisciplinarias.

Las plantas constituyen un atractivo sustrato para el desarrollo de enfermedades y plagas que son ocasionadas por innumerables parásitos, desde virus y bacterias hasta hongos, gusanos e insectos, entre otros posibles agentes patógenos biológicos. Junto con diversos factores físicos y químicos ambientales, los parásitos pueden ocasionar lesiones y hasta la muerte de las plantas. Los hongos fitopatógenos son los parásitos más frecuentes y numerosos entre los diversos microorganismos que causan enfermedades en las plantas. De manera que los fitopatólogos necesitan prestar especial atención al estudio de la micología y desarrollar 
proyectos de investigación para el control de los hongos que atacan a las plantas, en particular a las especies cultivadas de importancia económica como los cereales, las leguminosas y los árboles frutales. Es tal la importancia del control de los hongos fitopatógenos, que se han establecido comisiones especiales e institutos de investigación para estudiarlos con un enfoque específico, por ejemplo, para el estudio de las enfermedades del maíz y del trigo, de la caña de azúcar y de las plantas de importancia forestal. Todas las plantas, tanto las silvestres como las comestibles, pueden ser atacadas por diversas especies de hongos y, por lo tanto, cada especie presenta una entidad de investigación fitopatológica (Finch y Finch, 1974; Agrios, 1985; Romero-Cova, 1988).

\section{Micología médica humana y veterinaria}

Estos dos extensos campos de la micología son estudiados por los médicos, en forma rutinaria, con el objeto de controlar y curar las enfermedades micóticas que se presentan en el hombre y en los animales domésticos. También es importante el conocimiento de los hongos que atacan a los animales que se propagan con fines industriales, por ejemplo, en criaderos de peces, siguiendo los procedimientos adecuados de la zootecnia, según la especie que se desea propagar y el ambiente en que ésta se desarrolla. En todos los casos mencionados, existe la posibilidad de que los hospederos sean atacados por hongos patógenos u oportunistas, en distintos grados de severidad; de manera que pueden presentarse micosis leves, de mediana intensidad, graves o mortales, según la especie y la virulencia de los hongos involucrados en los procesos patológicos. Este fenómeno también depende de la respuesta inmunitaria de los hospederos afectados. Muchas veces la virulencia de estos hongos está asociada al dimorfismo, es decir a la capacidad que dichos hongos tienen de crecer como levadura o en forma micelial. Investigaciones sobre este fenómeno deben realizarse con más frecuencia para poder llegar a comprender las causas de la patogénesis de los hongos que atacan al hombre y a los animales.

Es interesante anotar que los casos de micosis han aumentado en las últimas décadas, debido al uso indiscriminado de los antibióticos de amplio espectro. Este uso favorece la capacidad invasiva de diversos hongos, incluso de algunos que son oportunistas y que sólo atacan en determinadas circunstancias, por ejemplo, en los casos terminales de sida en los que es frecuente la candidiasis ocasionada por Candida albicans. Las investigaciones que se realicen en el futuro sobre estos importantes fenómenos de la micología médica pueden ser de gran utilidad para lograr el control de las enfermedades micóticas (Howard, 1983; Bonifaz, 1990).

\section{DISCUSIÓN Y CONCLUSIONES}

De acuerdo a las anteriores explicaciones, puede comprenderse que el estudio de los hongos es importante en muy diversos campos de la ciencia en los que siempre está abierta la posibilidad de desarrollar investigaciones con múltiples enfoques básicos y aplicados, considerando que los hongos desempeñan una función esencial en el equilibrio ecológico de la naturaleza y en las relaciones íntimas que tienen con las plantas y los animales y en las actividades humanas. Los hongos y el hombre han convivido desde que éste apareció en la Tierra, siguiendo una interacción tanto en el sentido benéfico como en el nocivo, pues aunque hay hongos que deterioran materiales útiles o que contaminan alimentos y hongos que ocasionan enfermedades a las plantas cultivadas, a los animales domésticos y al hombre mismo, hay también numerosos hongos que por la versatilidad de su actividad química pueden ser utilizados en la agricultura y en la industria empleando con frecuencia complicados procedimientos biotecnológicos que sólo podrán ser superados en el futuro mediante el desarrollo de investigaciones diseñadas con una proyección bien definida en beneficio de la humanidad (Gray, 1959; Christensen, 1965). Por otra parte, la investigación micológica es también interesante si sólo se pretende conocer la complicada biología de los hongos en sus diversas manifestaciones.

Puede concluirse que las áreas de estudio de la micología ofrecen infinitas posibilidades para realizar investigaciones que seguramente brindarán grandes sorpresas a medida que salgan a la luz tanto los resultados esperados como otros sorpresivos. En México, así como en otros países en desarrollo, estas posibilidades son aun mayores que las de países situados en la vanguardia de la ciencia y la tecnología, aunque también en éstos son cada vez más numerosos los proyectos de trabajos en todos los campos de las investigaciones micológicas. Según esto, el esfuerzo para lograr resultados importantes en la investigación micológica debe ser cada vez mayor en los países subdesarrollados para alcanzar un mejor equilibrio con el desarrollo científico de los países que han progresado más en este tipo de investigación.

\section{LITERATURA CITADA}

Ainsworth GC, Hawksworth DL, Kirk PM, Sutton BC, Pegler DN. 1994. Ainsworth and Bisby's dictionary of the fungi. 8th Ed. CAB International.

Ainsworth GC, Sussman AS, edrs. 1965. The fungi: an advanced treatise. Vol.1, The Fungal Cell.

Alexopolus CJ, Mims CW. 1985. Introducción a la micología. Omega, Barcelona.

Agrios GN. 1985. Fitopatología. Limusa, México.

Bonifaz A. 1990. Micología médica básica. Ed. Méndez Cervantes.

Buller AHR. 1909-1950. Researches on fungi. 7 vols.; vols. 1-6, Longman, Green, Londres; vol. 7, University Of Toronto Press, Toronto.

Chang ST, Hayes WA. 1978. The biology and cultivation of edible mushrooms. Academic Press, Nueva York.

Christensen CM. 1965. The molds and man. An introduction to the Fungi. 3rd. ed. McGraw Hill Book Company.

Christensen CM. 1975. Molds, mushrooms and mycotoxins. University of Minnesota Press. Mineapolis.

Finch HC, Finch AN. 1974. Los hongos comunes que atacan cultivos en América Latina. Trillas, México.

Fincham JRS, Day PR, Redford A. 1979. Fungal genetics. 4th. ed. Blackwell, Londres. 
Gray WD. 1959. The relation of fungi to human affairs. Henry Holt, Nueva York.

Hawksworth DL, Sutton BC, Ainsworth GC. 1983. Ainsworth and Bisby's Dictionary of the Fungi. 7th. ed. Commonwealth Mycological Institute, Kew, Surrey.

Herrera T, Ulloa M. 1990. El reino de los hongos: micología básica y aplicada. UNAM-Fondo de Cultura Económica, México.

Howard DN, ed. 1983. Fungi pathogenic for humans and animals. Marcel Dekker, Nueva York.

Kohlmeyer J, Kohlmeyer E. 1979. Marine mycology. The higher fungi. Academic Press, Nueva York.

Marks GC, Kozlowski TT (Edrs.). 1973. Ectomycorrhizae -their ecology and physiology. Academic Press, Nueva York.

Martín D, Quirarte M, Soto C, Salmones D, Guzmán G. 1984.
Perspectivas sobre el cultivo de hongos comestibles en residuos agro-industriales en México. Bol. Soc. Mex. Mic. 19:207.

Raper JR, Flexer AS. 1971. Mating systems and evolution of the Basidiomycetes. The University of Tennessee Press, Nashville, 149-176.

Robinson PM. 1967. Ecology of fungi. The English University Press.

Robinson PM. 1978. Practical fungal physiology. John Wiley, Nueva York.

Romero-Cova S. 1988. Hongos fitopatógenos. Universidad Autónoma de Chapingo, México.

Smith G. 1960. An introduction to industrial mycology. Edward Arnold, Londres.

Talbot PHB. 1971. Principles of fungal taxonomy. St. Martin's Press, Nueva York. 